\title{
Climatology characterization of equatorial plasma bubbles using GPS data
}

\author{
Sergio Magdaleno ${ }^{1, *}$, Miguel Herraiz ${ }^{2,3}$, David Altadill ${ }^{4}$, and Benito A. de la Morena ${ }^{5}$ \\ 1 ESSP SAS, Torrejón de Ardoz, 28850, Spain \\ ${ }^{*}$ Corresponding author: smactor@gmail.com \\ 2 Department of Physics of the Earth, Astronomy and Astrophysics I, University Complutense, 28040 Madrid, Spain \\ 3 Institute of Geosciences, UCM - CSIC, 28040 Madrid, Spain \\ 4 Ebro Observatory (OE), CSIC - Universitat Ramon Llull, 43520 Roquetes, Spain \\ 5 Atmospheric Sounding Station El Arenosillo, INTA, 21130 Huelva, Spain
}

Received 25 January 2016 / Accepted 19 November 2016

\begin{abstract}
The climatology of equatorial plasma bubbles (EPBs) for the period 1998-2008 was studied using slant total electron content (sTEC) derived from global positioning system (GPS) data. The sTEC values were calculated from data measured at 67 International GNSS Service (IGS) stations distributed worldwide around the geomagnetic equator and embracing the region of the ionospheric equatorial anomaly (IEA). EPBs and their characteristics were obtained using the Ionospheric Bubble Seeker (IBS) application, which detects and distinguishes sTEC depletions associated with EPBs. This technique bases its analysis on the time variation of the sTEC and on the population variance of this time variation. IBS finds an EPB by default when an sTEC depletion is greater than 5 TEC units (TECu). The analysis of the spatial behavior shows that the largest rate of EPB takes place at the equator and in the South America-Africa sector, while their occurrence decreases as the distance from the magnetic equator increases. The depth and duration of the sTEC depletions also maximize at the equator and in the South America-Africa sector and weaken departing from the equator. The results of the temporal analysis for the data of the IGS stations located in AREQ, NKLG, IISC, and GUAM indicate that the greatest rate of EPB occurrence is observed for high solar activity.
\end{abstract}

Key words. Ionosphere (equatorial) - Total electron content - Aeronomy - Plasma physics - Remote sensing

\section{Introduction}

Ionospheric irregularities are defined as ionospheric regions with electron density clearly different from the background volume. They are the plasma expression of complex physical processes that can produce scintillation of the electromagnetic signals crossing the ionosphere and spread $\mathrm{F}$ in the equatorial ionograms. Density variations are defined as positive or negative if the anomaly with respect to the background shows an increase or a decrease in the number of electrons, respectively. Positive anomalies, known as "blobs", were first reported by Oya et al. (1986) and Watanabe \& Oya (1986) and have been studied less than the negative ones, known as "bubbles", that were observed and studied earlier (Woodman \& La Hoz 1976) and had received much more attention. The formation of the bubbles is very rapid due to very different and complex physical phenomena. The bubbles can adopt variable shapes and their size ranges up to seven orders of magnitude, from centimeters to hundreds of kilometers. Their duration can vary between minutes and several hours. The most interesting anomalies of this type correspond to depletions of the electronic density that manifest mainly at equatorial latitudes and that are known as Equatorial Plasma Bubbles (EPBs). These depletions are the result of the nonlinear evolution of the generalized Rayleigh-Taylor (R-T) instability in the post-sunset ionosphere (Kelley 1989) and can report densities more than two orders of magnitude lower than the background density. EPBs are often associated with Equatorial Spread F (ESF) observed in the ionograms.
The EPBs are usually formed in the bottomside $\mathrm{F}$ region, they rise to high altitudes and propagate eastward. Although EPBs are mainly equatorial phenomena, they can extend to higher latitudes along the magnetic field lines with a clearly defined structure that moves to consider them as "well-formed plasma density depletions" (Haaser et al. 2012).

The occurrence of bubbles is closely related to the scintillation phenomenon that strongly affects the performance of radio navigation and telecommunication systems and these plasma irregularities have received increasing attention over the last four decades. Bubbles have been studied with ground-based instruments such as ionosondes, radar, and airglow analyzers, and with satellite in situ instruments. The information derived from Global Navigation Satellite System (GNSS) records has played a significant role in ionospheric research since the late 1990s. The understanding of the physical phenomena leading to the formation of bubbles has gradually been established thanks to the contributions of, among others, Haerendel (1973), Basu \& Kelly (1977), Tsunoda et al. (1982), Sultan (1996), and Eccles (1998a, 1998b). These studies were done thanks to progress in the knowledge of the spatial and temporal distributions of the phenomenon. The pioneering global morphology of ionospheric scintillations was accomplished by Aarons (1982), who used ground-based measurements. Soon after, Maruyama \& Matuura (1984) presented the longitudinal variability of the annual occurrence of EPBs based on data from the Ionosphere Sounding Satellite (ISS) for 1978-1980. In turn, the retarding potential analyzer aboard the Atmosphere Explorer-E (AE-E) 
Table 1. List of the stations used and the corresponding geographical latitudes and longitudes. The number of days analyzed in each year and for each station, and the total number of days analyzed in each year are also included.

\begin{tabular}{|c|c|c|c|c|c|c|c|c|c|c|c|c|c|}
\hline \multirow[t]{2}{*}{ Station } & \multirow{2}{*}{$\begin{array}{l}\text { Geographical } \\
\text { longitude }\end{array}$} & \multirow{2}{*}{$\begin{array}{l}\text { Geographical } \\
\text { latitude }\end{array}$} & \multicolumn{11}{|c|}{ Number of days analyzed } \\
\hline & & & 1998 & 1999 & 2000 & 2001 & 2002 & 2003 & 2004 & 2005 & 2006 & 2007 & 2008 \\
\hline ADIS & 38.7663 & 9.0351 & & & & & & & & & & 139 & 281 \\
\hline AIRA & 130.5996 & 31.8241 & 289 & 338 & & & 352 & 361 & & & 344 & 359 & \\
\hline AREQ & -71.4928 & -16.4655 & 358 & 299 & 282 & 337 & 193 & 342 & 261 & 358 & 252 & 358 & 362 \\
\hline $\mathrm{ASC} 1$ & -14.4121 & -7.9512 & 346 & 312 & 269 & 19 & 287 & 333 & 170 & 54 & 37 & 9 & \\
\hline BAKO & 106.85 & -6.49 & 235 & 261 & 231 & 276 & 277 & 341 & 315 & 313 & 325 & 285 & 344 \\
\hline BAN2 & 77.51 & 12.95 & & & & & 29 & 359 & 321 & 235 & 206 & 256 & 275 \\
\hline BHR1 & 50.6081 & 26.2091 & & 262 & 309 & 305 & 355 & 363 & 336 & 338 & 347 & 357 & 327 \\
\hline BHR2 & 50.6081 & 26.2091 & & 9 & & & & & & 72 & 345 & 354 & 326 \\
\hline BOGT & -74.0809 & 4.6401 & 301 & 210 & & & 214 & 355 & & & 335 & 313 & \\
\hline BRAZ & -47.8778 & -15.9474 & 125 & 318 & 279 & 295 & 338 & 357 & 343 & 333 & 314 & 347 & 344 \\
\hline BRFT & -38.4255 & -3.8774 & & & & & & & & 107 & 342 & 331 & \\
\hline BUE1 & -58.5193 & -34.5737 & & 259 & & & 358 & 362 & & & 346 & 356 & \\
\hline CCJ2 & 142.195 & 27.0675 & & & & & & & & & & & 209 \\
\hline CFAG & -68.2326 & -31.6022 & & & 336 & 352 & 326 & 122 & 84 & 40 & 344 & 354 & 361 \\
\hline CHPI & -44.9852 & -22.6871 & & & & & & 141 & 310 & 237 & 344 & 353 & 338 \\
\hline CNMR & 145.7431 & 15.2297 & & & & & & 128 & 279 & 256 & 369 & 28 & 242 \\
\hline CONZ & -73.0255 & -36.8438 & & & & & & 342 & & & 322 & 356 & \\
\hline COPO & -70.3382 & -27.3845 & & & & & 137 & 270 & & & 270 & 248 & \\
\hline CUSV & 100.5339 & 13.7359 & & & & & & & & & & & 63 \\
\hline DAKA & -17.4648 & 14.685 & & & & & 191 & 258 & 158 & 186 & 181 & 41 & \\
\hline GLPS & -90.3037 & -0.743 & & & & & & 336 & 261 & 288 & 312 & 330 & 131 \\
\hline GMAS & -15.4676 & 27.7648 & & & & & 80 & 328 & 318 & 322 & 312 & 337 & 319 \\
\hline GMSD & 131.0156 & 30.5564 & & & & & & 11 & & & 255 & 317 & \\
\hline GUAM & 144.8683 & 13.5893 & 343 & 327 & 213 & 296 & 297 & 341 & 210 & 331 & 320 & 336 & 325 \\
\hline GUUG & 144.8027 & 13.4332 & & & & & & 90 & 287 & 302 & 322 & 258 & 236 \\
\hline HALY & 36.0999 & 29.1389 & & & & & & & 28 & 91 & 156 & 235 & \\
\hline HNLC & -157.8645 & 21.3033 & & 321 & & & & 329 & & & 313 & 220 & \\
\hline HYDE & 78.5509 & 17.4173 & & & & & 50 & 300 & 312 & 293 & 322 & 308 & 306 \\
\hline IISC & 77.5704 & 13.0212 & 283 & 289 & 27 & 118 & 306 & 309 & 287 & 306 & 313 & 324 & 159 \\
\hline IQQE & -70.1317 & -20.2735 & & & & & 140 & 194 & & & 38 & & \\
\hline ISPA & -109.3444 & -27.125 & & & & & & & & & 321 & 279 & \\
\hline KOKB & -159.6649 & 22.1263 & 352 & 326 & & & 205 & 315 & & & 324 & 337 & \\
\hline KOUR & -52.806 & 5.2522 & 310 & 226 & 289 & 264 & 308 & 297 & 349 & 363 & 305 & 320 & \\
\hline KUNM & 102.7972 & 25.0295 & 36 & 255 & 52 & 94 & 303 & 289 & 286 & 299 & 299 & 325 & 264 \\
\hline KWJ1 & 167.7302 & 8.7222 & 350 & 280 & 2 & 10 & 38 & & & & & & \\
\hline LAE1 & 146.9932 & -6.6737 & & & & 348 & 307 & 294 & 312 & 302 & 148 & & \\
\hline LHAS & 91.104 & 29.6573 & 309 & 228 & 225 & 281 & 327 & 295 & 247 & 227 & 251 & 28 & \\
\hline MALD & 73.5263 & 4.1887 & & 104 & 307 & 220 & 141 & 142 & 86 & 260 & 36 & & \\
\hline MANA & -86.2487 & 12.1491 & & & 162 & 285 & 330 & 279 & 219 & 228 & 198 & 243 & 262 \\
\hline MAS1 & -15.6333 & 27.7637 & 345 & 157 & 270 & 343 & 334 & 298 & 290 & 323 & 297 & 324 & 324 \\
\hline MAUI & -156.257 & 20.7067 & & 288 & & & 325 & 293 & & & 282 & 296 & \\
\hline MBAR & 30.7379 & -0.6015 & & & & 133 & 148 & 50 & 229 & 365 & 262 & 220 & \\
\hline MCIL & 153.9787 & 24.2901 & & & & & 21 & 280 & 320 & 242 & 237 & 321 & 174 \\
\hline MKEA & -155.4563 & 19.8014 & 312 & 253 & 220 & 286 & 333 & 305 & 229 & 309 & 292 & 322 & 266 \\
\hline MSKU & 13.552 & -1.6312 & & & & 187 & 253 & 100 & 61 & 324 & 283 & 17 & \\
\hline NAMA & 42.0446 & 19.2114 & & & & & & & 18 & 80 & & & \\
\hline NKLG & 9.6721 & 0.3539 & & & 247 & 345 & 333 & 309 & 286 & 317 & 265 & 290 & 310 \\
\hline NTUS & 103.6799 & 1.3458 & 243 & 199 & 109 & 182 & 241 & 303 & 319 & 314 & 222 & 144 & 305 \\
\hline NURK & 30.0899 & -1.9446 & & & & & & & & & & & 25 \\
\hline PIMO & 121.0777 & 14.6357 & & 191 & 7 & 40 & 183 & 302 & 154 & 151 & 288 & 300 & 301 \\
\hline POVE & -63.8963 & -8.7093 & & & & & & & & & & & 79 \\
\hline QUI1 & -78.4936 & -0.2152 & & & & & 3 & 18 & 338 & 339 & 292 & 299 & 327 \\
\hline QUI2 & -78.4936 & -0.2152 & & & & & & & & 72 & 292 & 301 & 361 \\
\hline $\mathrm{RCMN}$ & 36.8938 & -1.2210 & & & & & & & & & & 226 & \\
\hline RIOP & -78.652 & -1.6506 & 39 & 188 & 13 & 12 & & & & & & 138 & 176 \\
\hline SAVO & -38.4323 & -12.9392 & & & & & & & & & & & 82 \\
\hline SEY1 & 55.4794 & -4.6737 & & 148 & 48 & 245 & 125 & 107 & 332 & 90 & 215 & 152 & \\
\hline SHAO & 121.2004 & 31.0996 & 332 & 198 & & & 290 & 172 & & & 103 & 77 & \\
\hline SLOR & -87.4365 & 13.4239 & & & 66 & 212 & 194 & & & & & & \\
\hline SOLA & 46.4006 & 24.9107 & & & & & & & 109 & 66 & & 124 & \\
\hline TCMS & 120.9874 & 24.798 & & & & & & & & & 283 & 273 & 165 \\
\hline TNML & 120.9874 & 24.798 & & & & & & & & & 282 & 257 & 226 \\
\hline TWTF & 121.1645 & 24.9536 & & & & 23 & 303 & 274 & 253 & 317 & 275 & 271 & 259 \\
\hline UNSA & -65.4076 & -24.7275 & & & 317 & 151 & 313 & 293 & 294 & 323 & 279 & 284 & 283 \\
\hline
\end{tabular}


Table 1. (continued)

\begin{tabular}{|c|c|c|c|c|c|c|c|c|c|c|c|c|c|}
\hline \multirow[t]{2}{*}{ Station } & \multirow{2}{*}{$\begin{array}{c}\text { Geographical } \\
\text { longitude }\end{array}$} & \multirow{2}{*}{$\begin{array}{c}\text { Geographical } \\
\text { latitude }\end{array}$} & \multicolumn{11}{|c|}{ Number of days analyzed } \\
\hline & & & 1998 & 1999 & 2000 & 2001 & 2002 & 2003 & 2004 & 2005 & 2006 & 2007 & 2008 \\
\hline WUHN & 114.3573 & 30.5317 & 343 & 298 & & & 321 & 297 & & & 281 & 272 & \\
\hline YIBL & 56.1123 & 22.1865 & & & & & & 113 & 209 & 130 & 225 & 177 & 168 \\
\hline YKRO & -5.2401 & 6.8706 & & 75 & & & & & 125 & & & & 84 \\
\hline \multicolumn{3}{|c|}{ Total days analyzed } & 5251 & 6619 & 4280 & 5659 & 10172 & 12097 & 9345 & 9903 & 14523 & 14126 & 9389 \\
\hline
\end{tabular}

obtained measurements at $300-400 \mathrm{~km}$ altitude and provided a global latitudinal and longitudinal distribution of the irregularities in density (Kil \& Heelis 1998). The use of polar-orbiting constellation to study the global climatology of EPBs started in 2001, initially for solar maximum years (Huang et al. 2001) and later for the full solar cycle 1989-2000 (Huang et al. 2002). Observations given by the same set of satellites DMSP (Defense Meteorological Satellite Program) gave the overall trends in seasonal averages of EPBs (Burke et al. 2004a). A very similar geographical distribution was obtained for the period March 1999-June 2004 by the first Republic of China satellite ROCSAT-1 (Kil et al. 2009). Joint analysis of DMSP and ROCSAT-1 measurements offered a new view of the longitudinal distribution of EPBs (Burke et al. 2004b). DMSP in situ information recorded in the period 1989-2004 revealed noticeable differences in the climatology of EPBs for maximum and minimum solar activity and the importance of local time influences (Gentile et al. 2006). These approaches were accompanied by the use of scintillation data and by the increasing relevance of GPS-derived information. The joint use of both kinds of data enabled improvements in the knowledge of the spatial and temporal distributions of EPBs at regional scales (Cervera \& Thomas 2006).

This paper aims to present the climatology of EPBs for the period 1998-2008 at the global scale using worldwide data recorded at 67 IGS stations located around the geomagnetic equator. For this purpose, EPBs were characterized using the Ionospheric Bubble Seeker (IBS) application (Magdaleno et al. 2012a). Section 2 presents the database used in this study and the configuration of the IBS to characterize the EPBs, Section 3 describes the main results achieved in this research, and the manuscript ends with a Summary and conclusion section.

\section{Data}

We selected data available from 67 stations of the International GNSS Service (IGS) (Dow et al. 2009) placed in the equatorial region around the geomagnetic equator and embracing the region of the ionospheric equatorial anomaly (IEA). These data consist of Receiver INdependent EXchange (RINEX) files that are available at the Crustal Dynamics Data Information System (CDDIS) data center (ftp://cddis.gsfc.nasa.gov). The stations and their data availability used in this study are listed in Table 1. Dates have been selected to cover the whole 23rd solar cycle. Figure 1 depicts the solar activity of this cycle, based on the yearly average sunspot number $R_{\mathrm{i}}$, obtained from the International Sunspot Numbers dataset in the NOAA website (http://www.ngdc.noaa.gov/stp/spaceweather.html).

The sTEC can be estimated by comparing the signals received from the two global positioning system (GPS) dual-frequency transmissions on L1 $(1.57542 \mathrm{GHz})$ and L2 $(1.22760 \mathrm{GHz})$. We used the combination of the

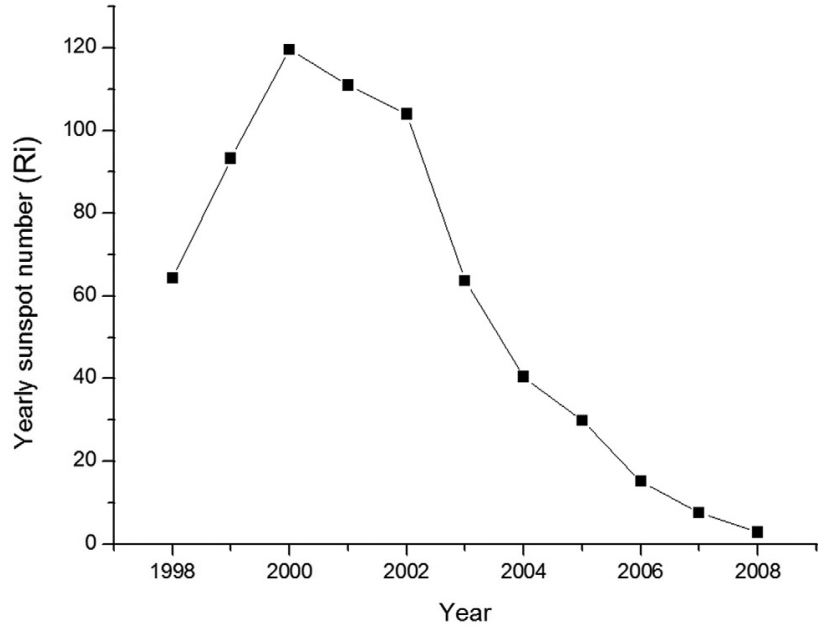

Fig. 1. Yearly average sunspot number, $R_{\mathrm{i}}$, for the $23 \mathrm{rd}$ solar cycle.

dual-frequency carrier-phase and code delay of GPS observations to obtain an ionospheric observable related to the sTEC (Ciraolo et al. 2007). The sTEC values were estimated from GPS observables obtained from RINEX files using the technique developed by Brunini \& Azpilicueta (2009). IGS orbit files, SP3, were used to estimate the elevation and azimuth of each line of sight. These files are also available at the CDDIS data center.

To detect and characterize the EPBs, we used the Ionospheric Bubble Seeker (IBS) application (Magdaleno et al. 2012a). This technique, based on the analysis of the sTEC arcs, identifies an EPB when an STEC depletion is located on the difference curve (original series minus calculated one). To detect a depletion, the IBS bases its analysis on the variation of the slope of the sTEC values and the population variance of these slope values. Four parameters are evaluated to characterize an EPB: the slope threshold (SLT), the variance threshold (VAT), the window size, and the depth threshold. The first three parameters determine the number of values that IBS uses to calculate the population variance. In addition, the IBS has an option to configure the minimum value of the elevation of the line of sight to discard errors due to the complex horizontal and vertical structure of the ionosphere (Vladimer et al. 1997).

This study considers the following parameters to find and characterize the EPBs: the window size equal to $600 \mathrm{~s}$, $\mathrm{SLT}=0.025 \mathrm{TECu} / \mathrm{s}$, VAT $=5 \times 10^{-5} \mathrm{TECu}^{2} / \mathrm{s}^{2}$, and minimum elevation $=25^{\circ}$. Moreover, a depth depletion threshold equal to $5 \mathrm{TECu}$ was selected to detect an EPB. IBS provides the number of depletions detected for each station and the number of days analyzed. In addition, a summary file is generated by the IBS that provides specific information about each EPB, such as the starting time, the ending time, and the 


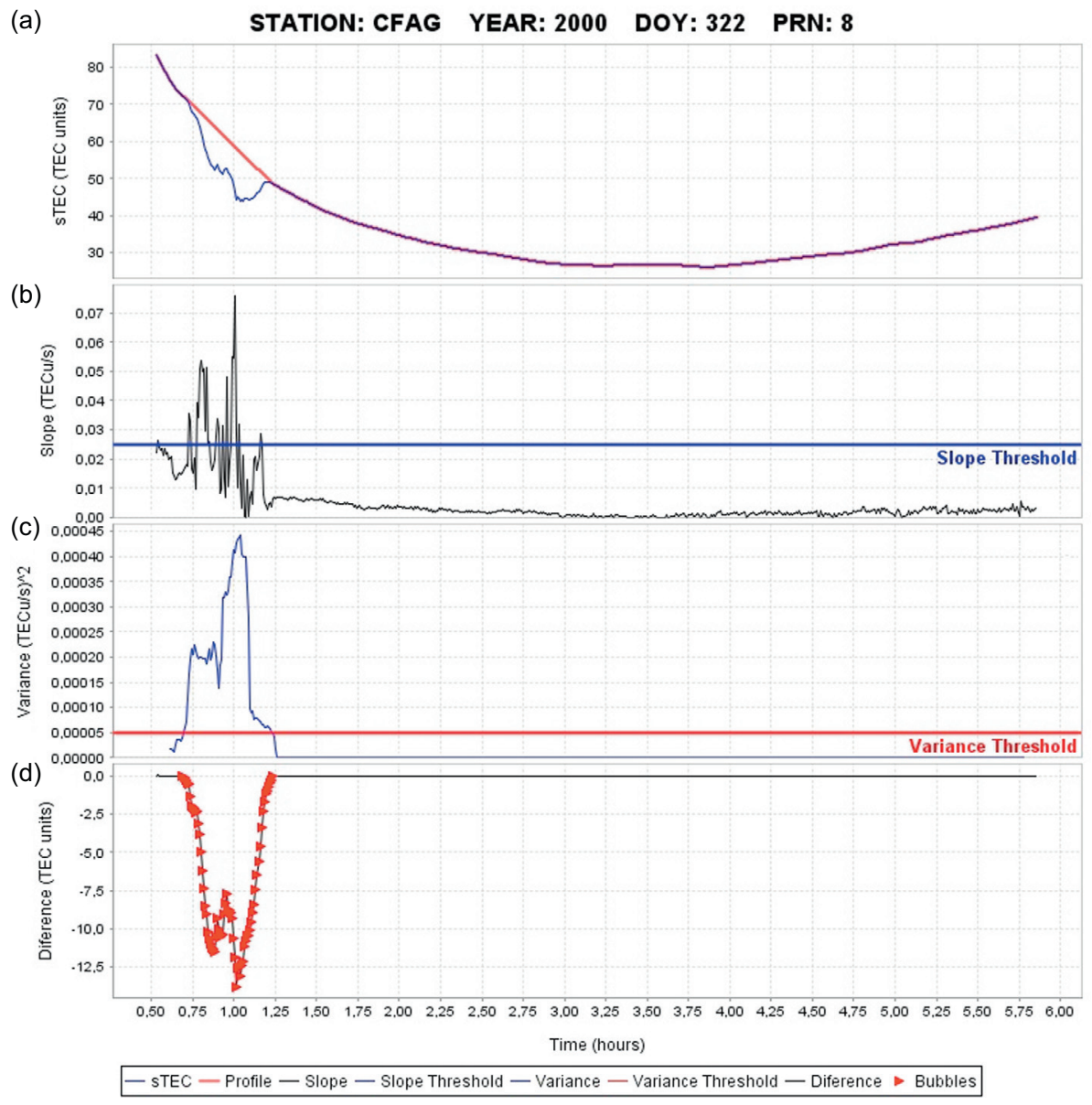

Fig. 2. Example of an IBS result for one sTEC arc from the CFAG station (PRN: 8, DOY: 322, YEAR: 2000). Panel (a) shows the real (black) and estimated (red) sTEC profile, panel (b) and (c) shows the sTEC gradient and variance, panel (d) shows the different between real and estimated sTEC profiles.

maximum difference between the original sTEC profile and the estimated one.

Figure 2 shows an example of an EPB detected by the IBS application at the CFAG station located in Argentina's West region on November 17, 2000. Figure 2a shows the original sTEC profile that is calculated from the RINEX file and the estimated profile, both in TECu, as a function of local time. Figures $2 \mathrm{~b}$ and $2 \mathrm{c}$ depict the slope of the sTEC profile and the variance, respectively, calculated by the IBS (their thresholds are indicated). Finally, Figure $2 \mathrm{~d}$ shows the difference curve, original series minus the calculated one. In this figure, the dots represent the points corresponding to the detected EPB. The first and the last dots are the starting and ending times, respectively. The difference between these values is the time duration of the depletion, the so-called "effective time", and it indicates the interval when the ray path from the satellite to the ground receiving station crosses the EPB. The depth of the depletion corresponds to the dot with the lowest value in the difference curve. Note that these values are negative.

\section{Results}

The spatial and temporal behaviors of the EPB are analyzed in this section. Section 3.1, presents the geographical distribution of the occurrence rate and the characteristics of the EPBs; i.e., their depth and effective time. The temporal behavior is discussed in Section 3.2, where the dependence of the rate of occurrence, of the depth, and of the effective time of EPBs on season and solar activity level is analyzed.

\subsection{Spatial distribution of EPBs}

To carry forward the analysis of the spatial distribution of EPBs, we used all 67 stations. This makes it possible to obtain good geographical distribution of the observations and to find the regional behavior of the occurrence and characteristics of the EPBs. Although the station network could not be considered very dense, it is noted that the results provided here are focused on the assessment of the climatological behavior and therefore, very high spatial resolution is not required. Moreover, the results agree with previous in situ data studies (e.g. Burke et al. 2004a, 2004b; Gentile et al. 2006), which confirm their reliability. All data available for the entire solar cycle 23 were analyzed to evaluate the aforementioned behavior under different solar activity conditions.

\subsubsection{Geographical distribution of the occurrence rate of the EPBS}

The origin and the development of the EPBs are linked to the magnetic latitudes. Figure 3 shows the geographical behavior of the occurrence rate of the EPBs, defined here as the number of EPBs detected per day analyzed. Figure 3 indicates that the larger values of the occurrence rate are observed close to 
S. Magdaleno et al.: Climatology characterization of equatorial plasma bubbles using GPS data
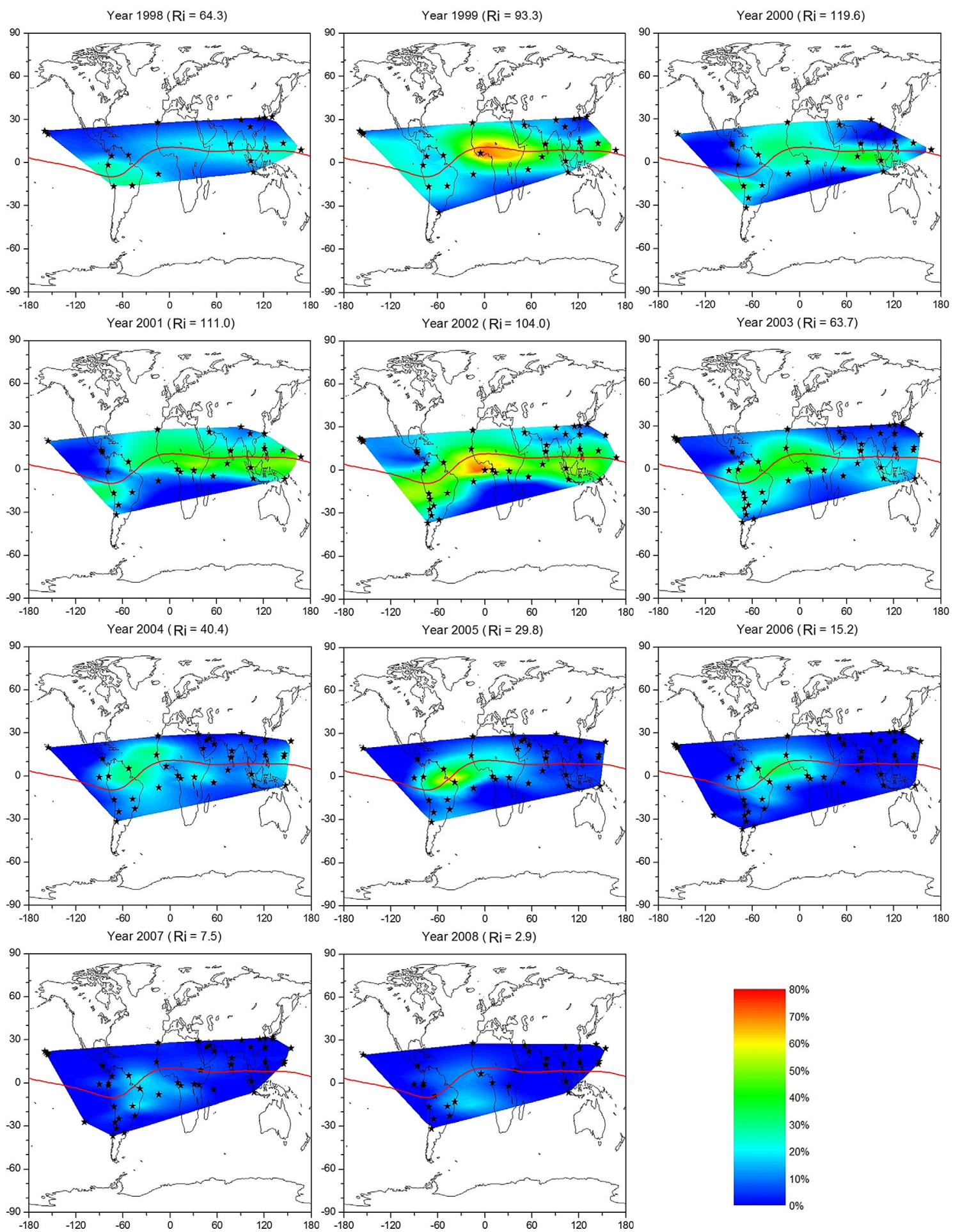

Fig. 3. Yearly EPB occurrence rate maps for the period 1998-2008. The black stars represent the location of the stations used for each map. The red line represents the magnetic equator.

the magnetic equator and these values decrease gradually as the distance from the magnetic equator increases. Moreover, the largest occurrence of EPBs is located in the AfricaAtlantic-South America sector. This behavior is observed for all solar activity levels. This dependence can reflect the characteristic variation of the equatorial magnetic field strength which influences the $V_{\mathrm{p}}=(E \times B) / B^{2}$ term in the R-T growth rate equation (Huang et al. 2001). The intensity of the Earth's magnetic field at the magnetic equator $\left(B_{\text {eq }}\right)$ presents longitudinal asymmetry. The largest values of the occurrence rate of EPBs are observed in the Africa-Atlantic-South America sector, where $B_{\text {eq }}$ is weak and the lowest values of occurrence rates of EPBs are located in the Indian-Pacific sector, where $B_{\mathrm{eq}}$ is strong. This result confirms that the occurrence of the EPB has a longitudinal dependence due to magnetic field intensity (Huang et al. 2001; Burke et al. 2004a).

The maximum values of the occurrence rate are observed at both sides of the magnetic equator. This behavior 

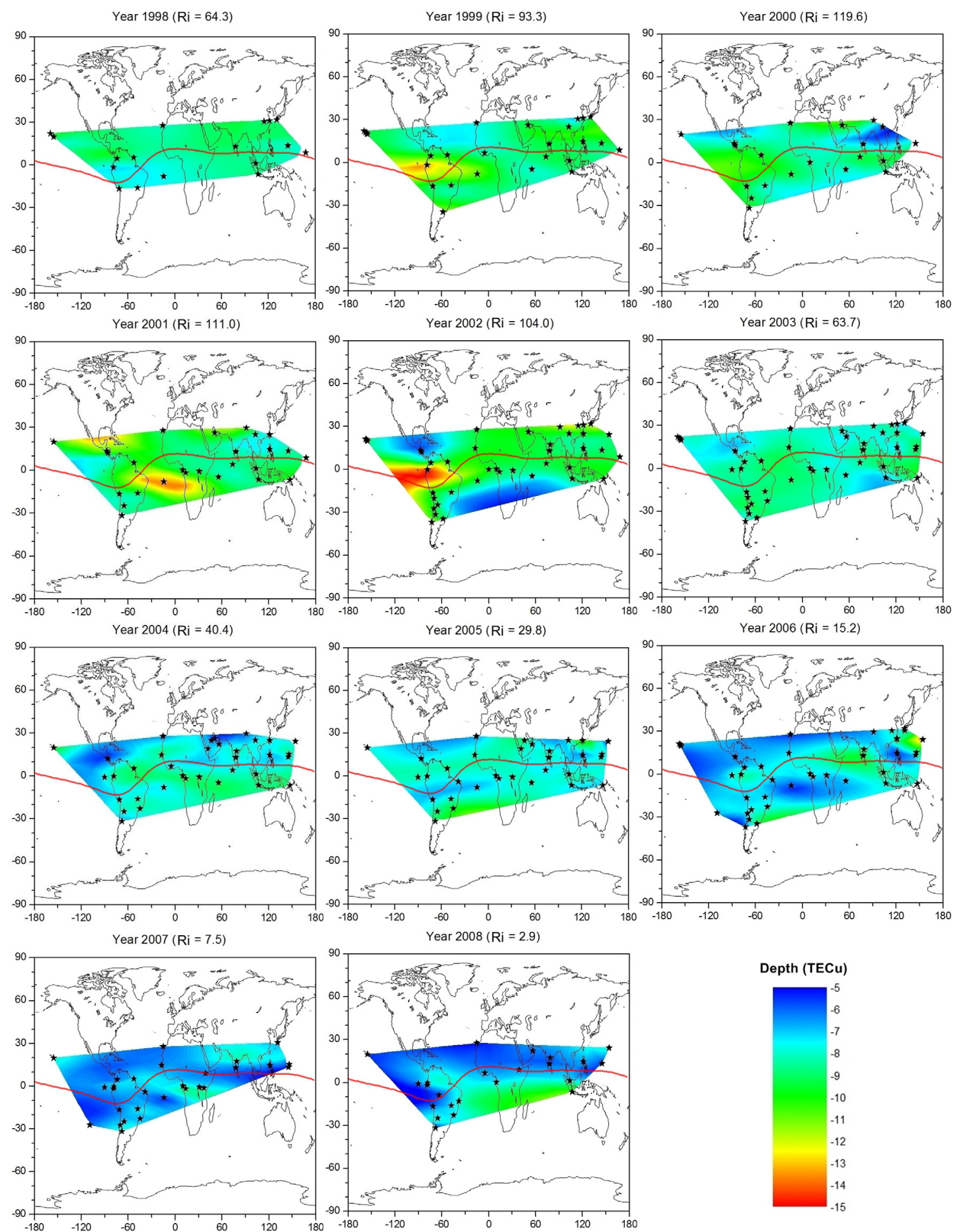

Fig. 4. Same as Figure 3 but for yearly EPB depth.

is observed during the whole solar cycle. The latter behavior is better defined in the African-Atlantic-South American sectors, where the occurrence rate of the EPB is larger.

The behavior of the EPBs clearly depends on both latitude and longitude. We have analyzed this behavior for four different longitude sectors: South America, Africa, Asia, and Pacific. This classification will be used further in the next section for temporal analysis.

\subsubsection{Geographical distribution of the characteristics of EPBS}

To examine the geographical behavior of the EPBs more closely, we focused on the analysis of two particular characteristics of EPBs:

- The depth of the EPBs, defined as the maximum difference between the sTEC profile and the calculated profile. 
S. Magdaleno et al.: Climatology characterization of equatorial plasma bubbles using GPS data
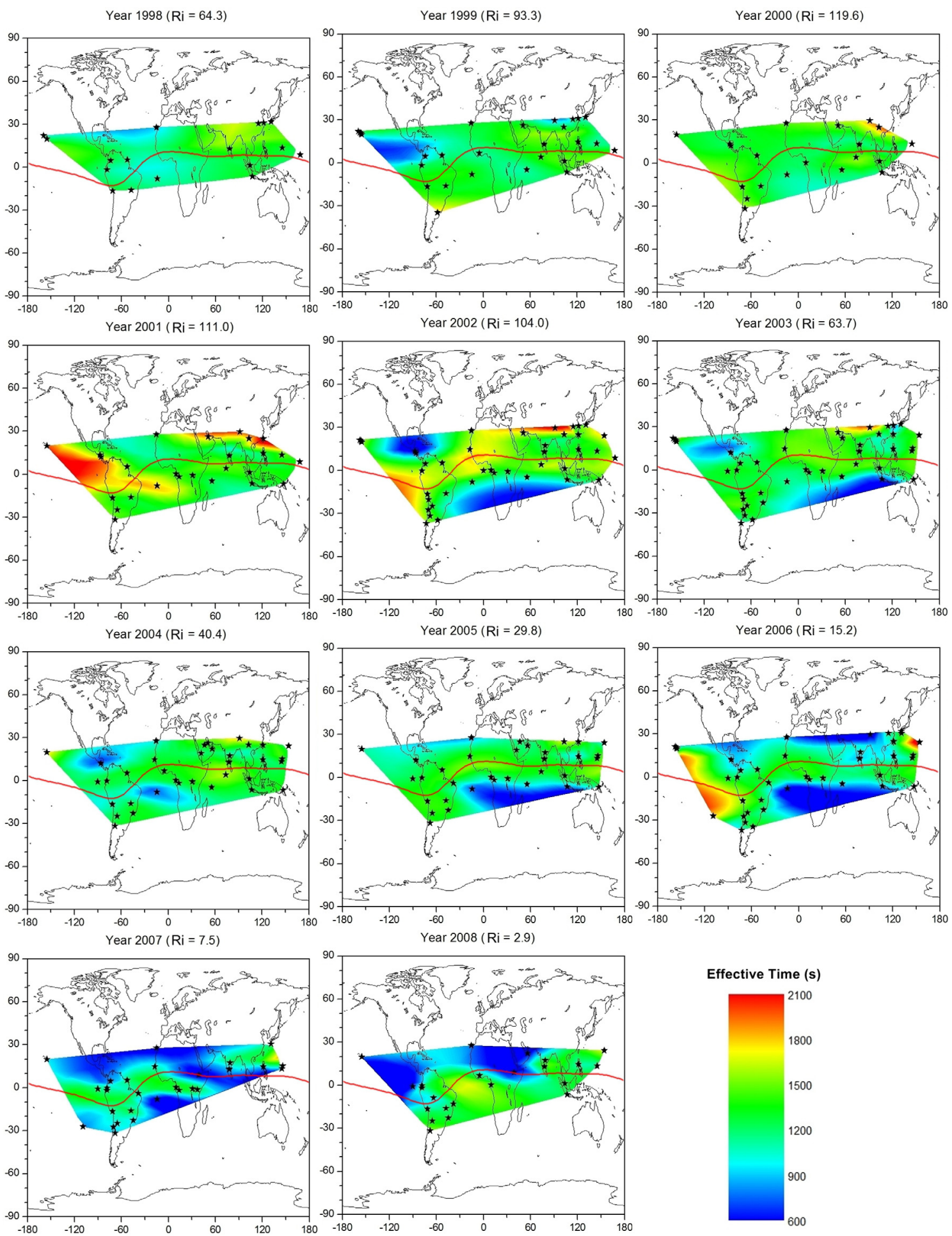

Fig. 5. Same as Figure 3 but for yearly EPB effective time.

- The effective time, i.e. the time interval when the ray path from the satellite to the ground receiving station crosses the EPB. This time interval is calculated as the difference between the times for the first point after ending the depletion and for the last point before starting the depletion. See the example plotted in Figure 2d.

Figure 4 depicts the geographical distribution of the depth of the EPBs. This figure shows an increase in the depth with increasing solar activity. The largest depth values occur in the South American sector. Figure 4 also shows a decrease in the values of the depth of the EPB with increasing magnetic latitude. This behavior agrees with the expected feature of the ionospheric plasma: EPBs are formed close to the magnetic equator due to $E \times B$ vertical drift and move to higher latitudes by the influence of the magnetic field (Burke et al. 2004a; Magdaleno et al. 2011, 2012b). As they advance, the recombination rate increases and the density difference generated by the EPB decreases. 


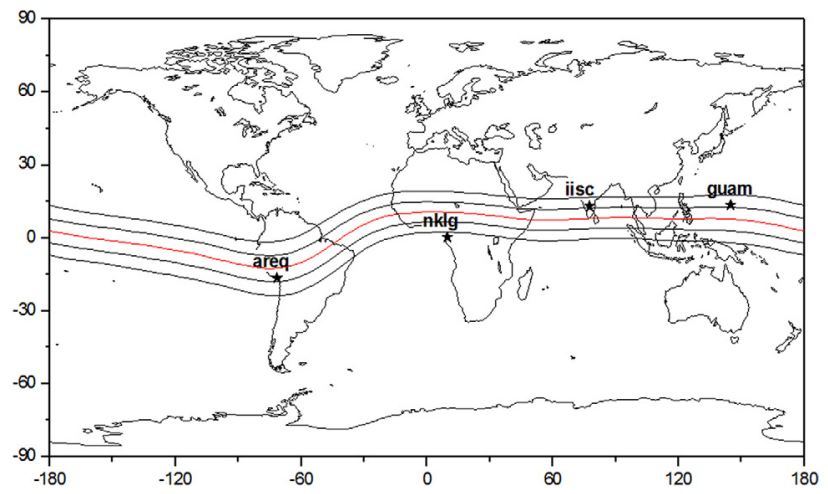

Fig. 6. Stations used for the temporal EPB distribution. Magnetic equator is plotted with the red line and stations are represented by black stars.

The geographical distribution of the effective time of the EPBs is plotted in Figure 5. As observed for the depth values, it shows that the larger effective times occur for higher solar activity levels. For this particular characteristic, the path of the satellite plays an important role. It is expected that close to the geographical equator, where the satellites are visible for longer, the effective time of the depletions should be larger than at latitudes far from the equator. In addition, the trajectory of the satellites influences the effective time of the EPBs: for the same EPB, the measurements recorded from signals of GPS satellites flying in the direction of the EPB's motion will report larger effective times than measurements from satellites flying in the opposite direction.

\subsection{Temporal distribution of the EPBs}

In this section, we analyze the temporal behavior of the occurrence rate of EPBs and its relation with the season and solar activity, as well as the temporal behavior of both EPB characteristics, depth and effective time. To carry forward this analysis, we focus on the data from four stations that are selected to cover four different longitudinal sectors, according to the results obtained in the previous subsection. Therefore, the AREQ, NKLG, IISC, and GUAM stations were selected for the South American, African, Asian, and Pacific sector, respectively. The distribution of these stations is plotted in Figure 6.

\subsubsection{Temporal behavior of the occurrence rate of EPBS}

First, we study the time dependence of the occurrence rate of the EPB for each of the four stations mentioned. For a given month, this rate is evaluated as the number of days of each month for which an EPB has been detected, divided by the total number of days analyzed for this particular month. The aim of this rate is to be an indicator of the probability of finding an EPB in order to assess EPB seasonal behavior. The occurrence rate of the EPBs as a function of season and solar activity is plotted in Figure 7. The solid black line indicates the two days per year when $\alpha=0^{\circ}$. By definition, $\alpha=\delta-\xi$ where $\delta$ and $\xi$ represent the declination and the terminator angles at the magnetic equator. As a function of Julian day, $D, \xi \approx 23.5^{\circ} \operatorname{Sin}(\pi(D-81.0) / 182.5)$. Figure 7 indicates that the occurrence of EPBs has an important dependence on solar activity, with larger occurrence rates of EPBs for higher solar activity levels and lower rates for lower solar activity. This temporal behavior was also reported in the
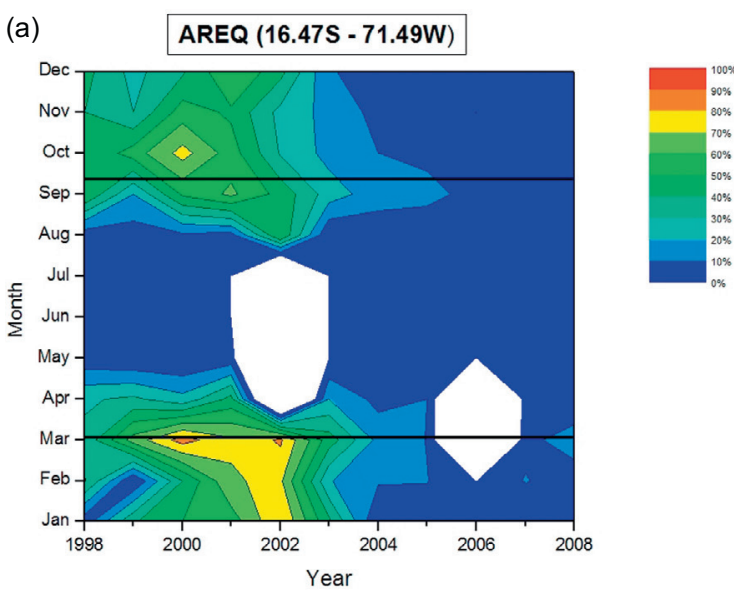

(b)
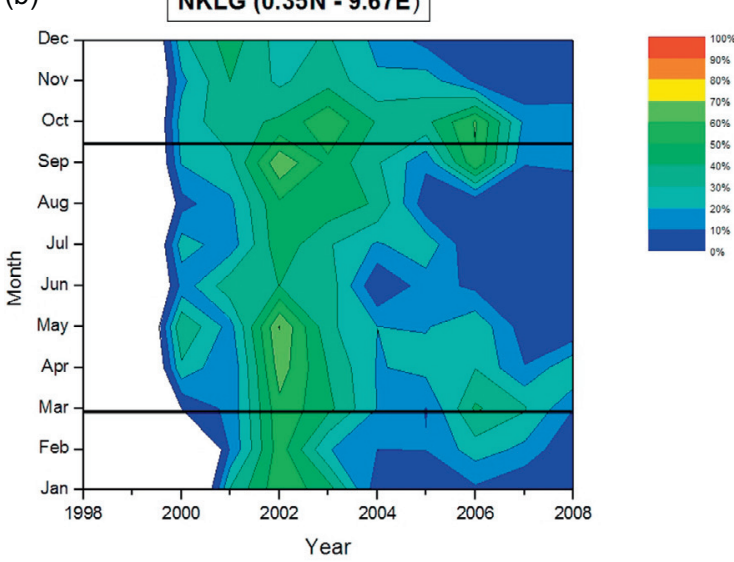

(c)
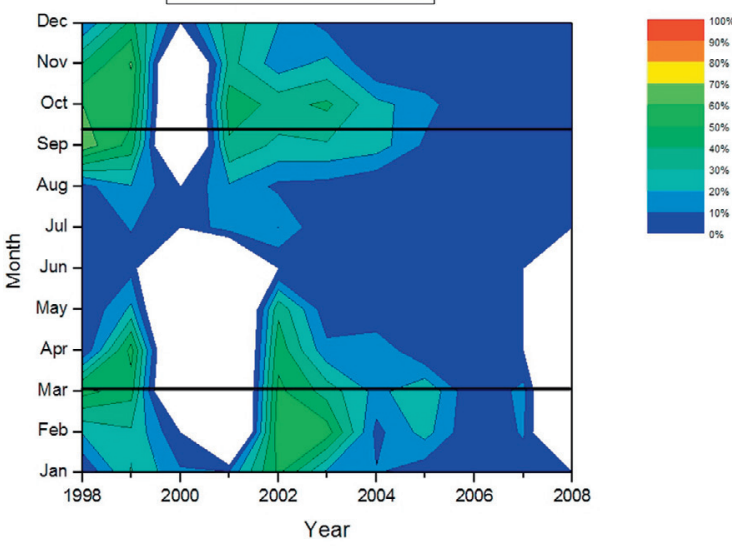

(d)

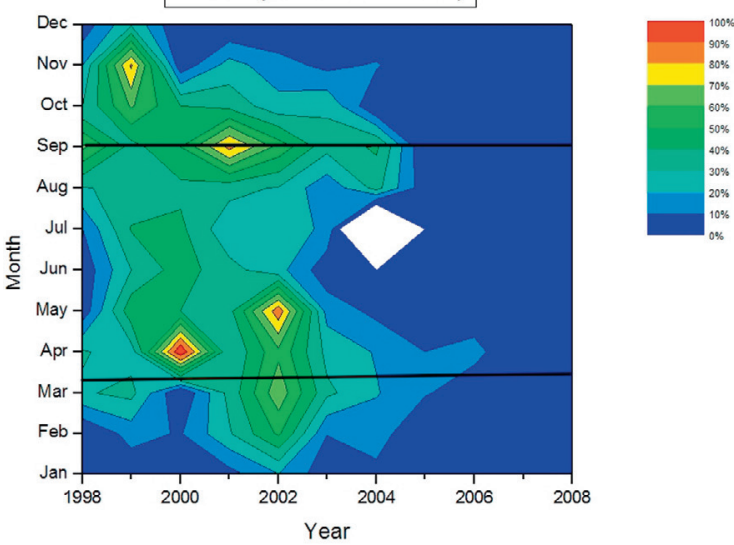

Fig. 7. Occurrence EPBs rate temporal distribution for IGS stations AREQ (a), NKLG (b), IISC (c), and GUAM (d). 
S. Magdaleno et al.: Climatology characterization of equatorial plasma bubbles using GPS data
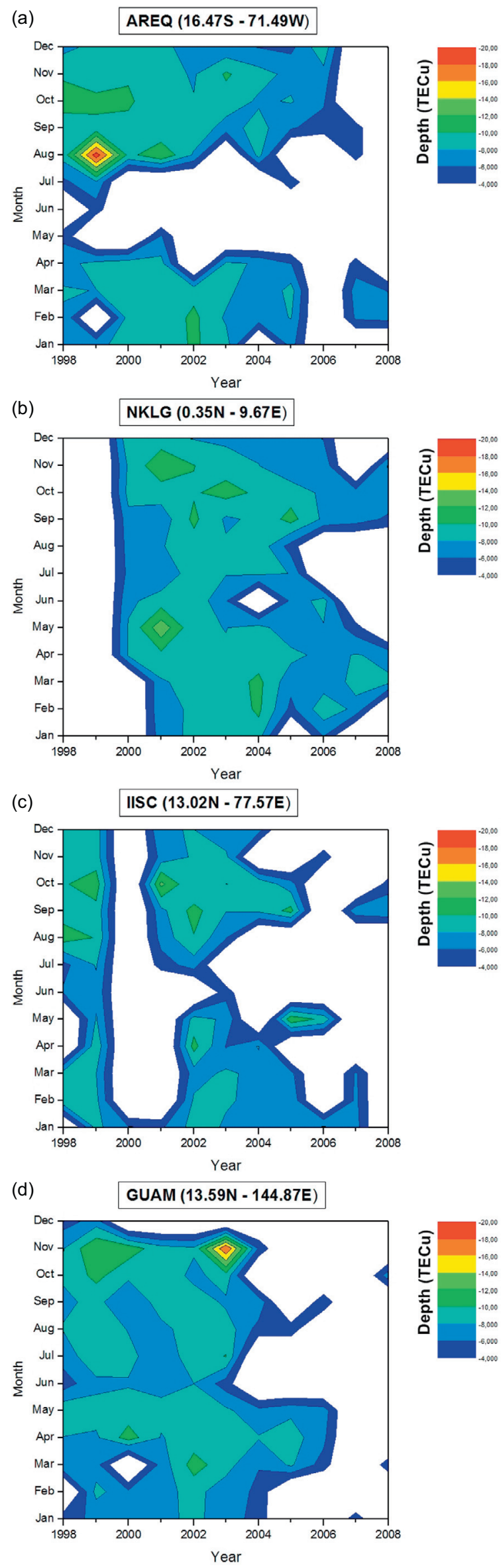

Fig. 8. Same as Figure 7 but for the EPB depth.
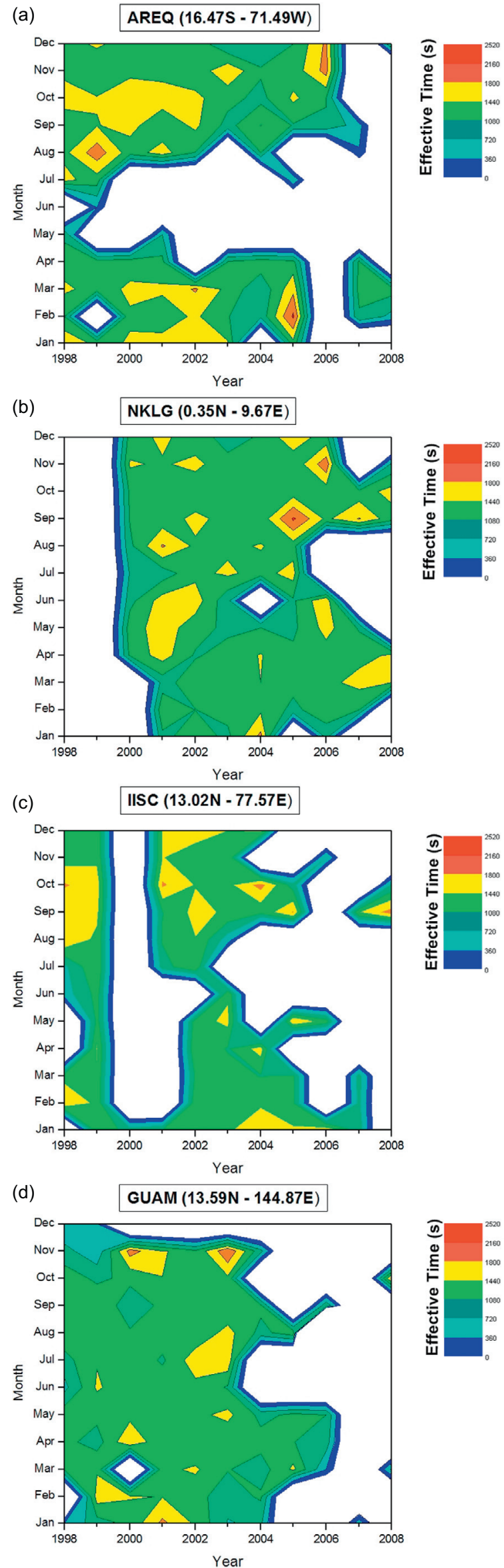

Fig. 9. Same as Figure 7 but for the EPB effective time. 
analysis for geographical dependence provided in the previous section. The results shown in Figure 7 also indicate that the seasonal behavior differs in different longitudinal sectors. The AREQ station, Figure $7 \mathrm{a}$, reports the maximum occurrence rate of EPBs during equinox months, while the presence of EPBs is low or null for the May-August period. The relative position of the geomagnetic equator and the line of the solar terminator can explain these results. In AREQ latitudes, the angle is close to perpendicular for September and April, so $E \times B$ vertical plasma drift produces its largest values. Magdaleno et al. (2012b) showed that the greatest values for the occurrence rates of EPBs are observed when the vertical plasma drift presents its greatest values. The results obtained here agree with the correlation presented in this study. Figure $7 \mathrm{~b}$ represents the behavior of the occurrence rate for the NKLG station. This station is located close to the geographic equator and reports EPB formation for the whole year, with larger values during the equinox months compared to solstices. This behavior agrees with the relative position between the geomagnetic equator and the terminator described above. It is remarkable that the presence of EPBs during low solar activity conditions is only evident in the African sector. Figure 7c shows the results for station IISC located in the Asian sector. IISC reports similar behavior in the occurrence rate of the EPBs to that observed in AREQ, with maximum values for equinox months. In addition, IISC shows a significant occurrence rate of EPBs from December to February, but the values of the rate, that is, the probability of an EPB forming, are low. Finally, Figure $7 \mathrm{~d}$ depicts the results for GUAM, located in the Pacific sector. The occurrence rate shows a significant probability of EPBs from February to November for this station. As observed for the other stations analyzed, the maximum occurrence rate of EPBs is observed during equinoxes. In addition, GUAM reports a significant probability of observing EPBs during the local summer months, as observed in the AREQ station, but in this case, from May to August.

\subsubsection{Temporal behavior of the characteristics of EPBS}

In this section, we analyze the behavior of the depth and the effective time of the EPBs with respect to season and solar activity. The behavior of the depth is presented in Figure 8 where, similarly to Figure 7 , the vertical axes of each plot correspond to the month number and the horizontal ones to the years analyzed. Figure 8a corresponds to the depth behavior obtained for the South American station, AREQ. This plot shows a correlation between the occurrence rate and the depth of the EPBs with maximum values for both parameters for the equinox months. Moreover, the depth of the EPBs decreases as the solar activity decreases. Figure $8 \mathrm{~b}$ corresponds to the NKLG station, located in the African sector and shows quite similar results to those of the AREQ station, a correlation between the occurrence rate and the depth of the EPBs. In this case, the depth of the EPBs shows a maximum value during equinoxes. Again, the values of the depth decrease as the solar activity does. Figure $8 \mathrm{c}$ shows the results for the IISC station located in the Asian sector. The maximum depth values of IISC are recorded in the equinox months and the depth values decrease with decreasing solar activity, as for the previous analysis. Finally, the results obtained for the depth behavior observed in the GUAM station, located in the Pacific sector, are depicted in Figure 8d. This station also reports the maximum depth values during equinoxes.

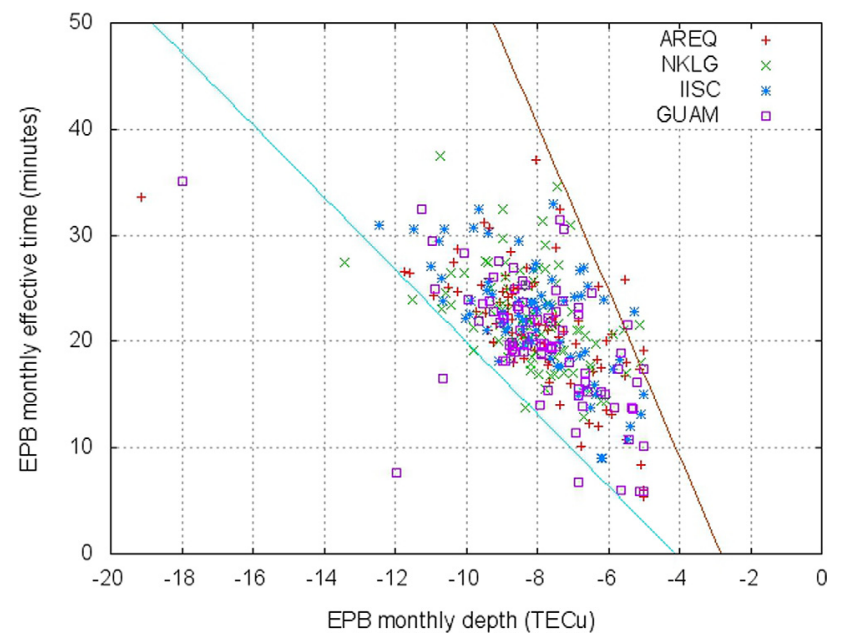

Fig. 10. EPB monthly effective time versus EPB depth values for the AREQ, NKLG, IISC, and GUAM stations. Solid lines bound the $95 \%$ of the Depth-Effective time values.

The relative position of the geomagnetic equator and the line of the solar terminator can explain these results. The formation of the pre-reversal enhancement (PRE) with the Rayleigh-Taylor instability provides favorable conditions for the generation of EPBs (Kelley 1989; Fejer et al. 1999). The EPB would be generated because the plasma from lower layers (with a plasma density much lower than the density of the F2 layer) uplifts to the F2 layer. As this uplift depends on $E \times B$ vertical plasma drift, the greater this value, the lower the plasma density uplifted closer to the maximum density, $\mathrm{NmF2}$, and therefore, the greater the depth of the EPB observed. In addition, this agrees with the results provided in Magdaleno et al. (2012b), where it is shown that the largest EPB depth values are found when the maximum values of the $\mathrm{NmF} / \mathrm{NmE}$ ratio are observed.

Figure 9 depicts the results of the temporal distribution of the effective time. The behavior of this characteristic presents a strong correlation with the behavior of the depth presented above. It is noticeable that the effective time of EPBs recorded in all four stations has very similar values for different sectors. By comparing the behavior of the effective time of EPBs with the depth or with the occurrence rate, it is clearly observed that larger effective times coincide with larger values of the other two characteristics. Note that the effective time behaves like a step function, with greatest values for equinox months and values at around 20-30 $\mathrm{min}$ for the rest of the year.

Finally, the correlation between the EPB depth and effective time was assessed. Figure 10 shows the EPB monthly effective time values for the four stations analyzed here (AREQ, NKLG, IISC, and GUAM) versus the EPB monthly depth values. From this figure, it could be concluded that although expected, there is no linear correlation between the effective time and the depth. However, the two solid lines show that there is a correlation between these values as for each effective time there are a limited set of values for the depth and vice versa.

\section{Summary and conclusion}

In this paper, we studied the spatial and temporal behaviors of EPBs for one solar cycle. For this purpose, we analyzed the 
TEC data estimated from 67 IGS stations distributed longitudinally around the geomagnetic equator. We focused the analysis on the 23rd solar activity cycle due to the large amount of available data. TEC values were deduced from RINEX files using the technique developed by Brunini \& Azpilicueta (2009). The IBS application (Magdaleno et al. 2012a) was used to detect the EPBs and compute their characteristics. This tool provides the number of detected EPBs, the number of days analyzed, and the depth and effective time of each EPB identified by the IBS.

This investigation focused the analysis on two aspects: the spatial and temporal behaviors of the EPBs. The occurrence rate of the EPBs and their two main characteristics, depth and effective time, were studied in each analysis.

The spatial analysis confirms that the behavior of the EPBs depends on latitude and longitude. In a global manner, the largest values for the occurrence rates of EPBs were recorded at the magnetic equator and these values decrease as the distance from the equator increases. The South America-Africa sector presents larger occurrence rates of EPBs than the other longitudinal sectors. According to the physical phenomena that can drive the EPBs, we should note that the largest values of vertical plasma drift, which at low latitudes is mainly driven by $E \times B$, are observed at the magnetic equator. This vertical drift uplifts the electron population from lower layers (i.e. the $E$ region and/or F1 layer) to the F2 layer and above, helping to generate vertical gradients of electron density, which in turn favors the generation of the Rayleigh-Taylor instability and the formation of EPBs. Thus, the larger the vertical drift and the electron density gradients, the higher the probability of EPB formation. Our results show that the highest values of EPB occurrence rates are observed at the magnetic equator where vertical plasma drift is expected to be the largest, confirming that close to the magnetic equator is the region where the highest probability for the generation of the EPBs is expected. Moreover, this is the region where the depth and duration (effective time) of the EPBs show the highest values. Therefore, the region with expected larger values of vertical electron density gradients presents larger values of depth and effective time of EPBs.

More detailed analysis is developed to evaluate the temporal behavior in different longitudinal sectors, South American, African, Indian, and Pacific sectors represented by the four stations AREQ, NKLG, IISC, and GUAM, respectively. The results of this analysis show that the occurrence rate of EPBs increases as solar activity increases. In addition, this temporal analysis shows that the seasonal behavior of the characteristics of the EPBs is different for different longitudinal sectors. We can speculate that the observed longitudinal dependence of the aforementioned characteristics is related to the relative angle between the line of the geomagnetic equator and the line of the solar terminator. Therefore, the largest occurrence rate of the EPBs is observed for equinox months. Moreover, both the AREQ and IISC stations present a significant occurrence rate of EPBs from November to February, while no EPBs are observed from June to August. However, the GUAM station reports the opposite behavior to that observed in AREQ and IISC: a significant occurrence rate of EPBs is shown from June to August, while no EPBs appear to exist from November to February. The depth and the effective time of the EPBs manifest a temporal behavior similar to that of the occurrence rate and they reach their highest values during the equinox months. The correlation analysis of these two parameters has shown that there is no linear correlation.
The present work offers a description of the worldwide distribution of EPBs and covers the whole 23rd solar cycle. These results can be useful to the system design of local or regional Satellite-Based Augmentation Systems (SBAS) located at equatorial latitudes such as the European SBAS (European Geostationary Navigation Overlay Service (EGNOS)), the Indian SBAS (GPS Aided Geo Augmented Navigation (GAGAN)), or the future African or South American SBAS.

Acknowledgements. D.A. thanks Spanish Ministry MINECO for supporting his work through project CTM2014-52182-C3-1-P. The authors would like to thank Dr. C. Brunini for his help and wish to express their gratitude to the International GNSS Service, the Crustal Dynamics Data Information System Data Center, and the National Oceanic and Atmospheric Administration for making the data available. The authors and editor thank two anonymous referees for their assistance in evaluating this paper.

\section{References}

Aarons, J. Global morphology of ionospheric scintillation. Proc. IEEE., 70, 360-378, 1982, DOI: 10.1109/PROC.1982.12314.

Basu, S., and M.C. Kelly. Review of equatorial scintillation phenomena in light of recent developments in the theory and measurement of equatorial irregularities. J. Atmos. Terr. Phys., 39, 1229-1247, 1977

Brunini, C., and F. Azpilicueta. Accuracy assessment of the GPSbased slant total electron content (sTEC). J. Geod., 83, 773-785, 2009, DOI: $10.1007 / \mathrm{s} 00190-008-0296-8$.

Burke, W.J., C.Y. Huang, L.C. Gentile, and L. Bauer. Seasonallongitudinal variability of equatorial plasma bubbles. Ann. Geophys., 22, 3089-3098, 2004a.

Burke, W.J., L.C. Gentile, C.Y. Huang, C.E. Valladares, and S.Y. Su. Longitudinal variability of equatorial plasma bubbles observed by DMSP and ROCSAT-1. J. Geophys. Res., 109, A12301, 2004b, DOI: 10.1029/2004JA010583.

Cervera, M.A., and R.M. Thomas. Latitudinal and temporal variation of equatorial ionospheric irregularities determined from GPS scintillation observations. Ann. Geophys., 24, 3329-3341, 2006.

Ciraolo, L., F. Azpilicueta, C. Brunini, A. Meza, and S.M. Radicella. Calibration errors on experimental slant total electron content determined with GPS. J. Geod., 81 (2), 111-120, 2007.

Dow, J.M., R.E. Neilan, and C. Rizos. The International GNSS Service in a changing landscape of Global Navigation Satellite Systems. J. Geod., 83, 191-198, 2009, DOI: $10.1007 / \mathrm{s} 00190-008-0300-3$.

Eccles, J.V. Modeling investigation of the evening prereversal enhancement of the zonal electric field in the equatorial ionosphere. J. Geophys. Res., 103 (A11), 26709-26719, 1998a.

Eccles, J.V. A simple model of low-latitude electric field. J. Geophys. Res., 103 (A11), 26699-26708, 1998 b.

Fejer, B.G., L. Scherliess, and E.R. de Paula. Effects of the vertical plasma drift velocity on the generation and evolution of equatorial spread F. J. Geophys. Res., 104, 19859-19869, 1999.

Gentile, L.C., W.J. Burke, and F.J. Rich. A global climatology for equatorial plasma bubbles in the topside ionosphere. Ann. Geophys., 24, 163, 2006.

Haaser, R.A., G.D. Earle, R.A. Heelis, J. Klenzing, R. Stoneback, W.R. Coley, and A.G. Burrell. Characteristics of low-latitude ionospheric depletions and enhancements during solar minimum. J. Geophys. Res., 117, A10305, 2012, DOI: $10.1029 / 2012 J A 017814$.

Haerendel, G. Results from barium cloud releases in the ionosphere and magnetosphere. Space Res., 13, 601-617, 1973.

Huang, C.Y., W.J. Burke, J.S. Machuzak, L.C. Gentile, and P.J. Sultan. DMSP observations of equatorial plasma bubbles in the topside ionosphere near solar maximum. J. Geophys. Res., 106, 8131, 2001 
Huang, C.Y., W.J. Burke, J.S. Machuzak, L.C. Gentile, and P.J. Sultan. Equatorial plasma bubbles observed by DMSP satellites during a full solar cycle: toward a global climatology. J. Geophys. Res., 107, 1434, 2002, DOI: 10.1029/2002JA009452.

Kelley, M.C. The Earth's ionosphere: plasma physics and electrodynamics. In: International Geophysics Series, vol. 43, Academic Press, San Diego, CA, 1989.

Kil, H., and R.A. Heelis. Global distribution of density irregularities in the equatorial ionosphere. J. Geophys. Res., 103, 407-417, 1998.

Kil, H., L.J. Paxton, and S.-J. Oh. Global bubble distribution seen from ROCSAT-1 and its association with the evening prereversal enhancement. J. Geophys. Res., 114, A06307, 2009, DOI: $10.1029 / 2008$ JA013672.

Magdaleno, S., S.M. Radicella, and M. Herraiz. Longitude characterization of GPS derived TEC depletions using a postprocessing automatic technique. In: Proceedings of 13th International Ionospheric Effects Symposium IES2011, 17-19 May, Alexandria, USA, 2011.

Magdaleno, S., M. Herraiz, and S.M. Radicella. Ionospheric bubble seeker: a Java application to detect and characterize ionospheric plasma depletion from GPS data. IEEE Trans. Geosci. Remote Sens., 50 (5), 1719-1727, 2012a, DOI: 10.1109/TGRS.2011.2168965.

Magdaleno, S., M. Herraiz, and B.A. de la Morena. Characterization of equatorial plasma depletions detected from derived GPS data in South America. J. Atmos. Sol. Terr. Phys., 74, 136-144, 2012b, DOI: $10.1016 /$ j.jastp.2011.10.014.

Maruyama, T., and N. Matuura. Longitudinal variability of annual changes in activity of Equatorial Spread $\mathrm{F}$ and plasma bubbles. J. Geophys. Res., 89, 10903-10912, 1984.

Oya, H., T. Takahashi, and S. Watanabe. Observation of low latitude ionosphere by the impedance probe on board the Hinotori satellite. J. Geomagn. Geoelectr., 38, 111-123, 1986.

Sultan, P.J. Linear theory and modelling of the Rayleigh-Taylor instability leading to the occurrence of Equatorial Spread F. J. Geophys. Res., 101, 26875-26801, 1996.

Tsunoda, R.T., R.C. Livingston, J.P. McClure, and W.B. Hanson. Equatorial plasma bubbles: vertically elongated wedges from the bottomside F layer. J. Geophys. Res., 87, 9171-9180, 1982.

Vladimer, J.A., M.C. Lee, P.H. Doherty, D.T. Decker, and D.N. Anderson. Comparisons of Topex and Global Positioning System total electron-content measurements at equatorial anomaly latitudes. Radio Sci., 32, 2209-2220, 1997.

Watanabe, S., and H. Oya. Occurrence characteristics of low latitude ionosphere irregularities observed by impedance probe on board Hinotori satellite. J. Geomagn. Geoelectr., 38, 125-149, 1986.

Woodman, R.F., and C. La Hoz. Radar observations of F region equatorial irregularities. J. Geophys. Res., 81, 5447-5466, 1976

Cite this article as: Magdaleno S, Herraiz M, Altadill D \& de la Morena B.A. Climatology characterization of equatorial plasma bubbles using GPS data. J. Space Weather Space Clim., 7, A3, 2017, DOI: 10.1051/swsc/2016039. 